\section{Ist Heuschnupfen wirklich chronisch?}

\author{
Spontane Remissionen der allergischen Rhinitis sind dem Kliniker \\ wohl bekannt. Eine Langzeitbeobachtung sollte jetzt klären, ob es \\ sich dabei um Raritäten handelt oder ob das Konzept der Chronizität \\ der allergischen Rhinitis in Frage gestellt werden muss.
}

$\mathrm{D}$ änische Forscher dokumentierten in den Jahren 1990 und 1998 die Daten von 734 Probanden. Die Teilnehmer gaben in einem Fragebogen Auskunft zu Atemwegserkrankungen und entsprechenden Symptomen. Zusätzlich wurde die Konzentration spezifischer IgE-Antikörper im Serum erfasst. Eine allergische Rhinitis lag definitionsgemäß vor, wenn in den letzten 12 Monaten Symptome bei Allergenexposition aufgetreten waren und die Konzentration des entsprechenden spezifischen $\operatorname{IgE}$ über $0,7 \mathrm{kU} / \mathrm{l}-$ also mindestens RAST-Klasse 2 - lag.

Insgesamt 257 der 734 Studienteilnehmer erfüllten zu Untersuchungsbe- ginn die Kriterien für das Vorliegen einer allergischen Rhinitis, 170 aufgrund einer Allergie gegen Birken-, Gras- oder Beifußpollen, 53 reagierten allergisch auf Katze oder Hund, 34 Probanden hatten eine Hausstaubmilbenallergie. Nach 8 Jahren waren insgesamt $17 \%$ der Patienten nach eigenen Angaben symptomfrei geworden. Bei den Pollenallergikern war die Quote mit 12\% am niedrigsten, bei den Tierhaarallergikern lag sie bei $19 \%$ und im Falle der Hausstauballergie sogar bei $38 \%$. Alle Patienten mit Remission zeigten gegenüber der ersten Untersuchung reduzierte Werte für das spezifische IgE, aber nur bei $22 \%$ war die Kon- zentration auf Werte kleiner als Klasse 2 gesunken. Somit waren zwar einige Teilnehmer der Studie in der zweiten Untersuchung symptomfrei, aber weiter sensibilisiert gegen das Allergen. Remissionen waren korreliert mit einem niedrigen spezifischen IgE zu Beginn der Studie. Die unterschiedlichen Remissionsraten führen die Autoren darauf zurück, dass eine Karenz bei Pollenallergie schwieriger zu erzielen ist als bei Tierhaar- oder Hausstaubmilbenallergie.

Fazit: Eine Remission der allergischen Rhinitis mit Symptomfreiheit und einer deutlich abgesunkenen Konzentration des spezifischen IgE ist nur selten zu beobachten - die Autoren sehen damit die Auffassung bestätigt, dass die allergische Rhinitis eine chronische Erkrankung darstellt.

Bodtger $U$ et al. Remission of allergic rhinitis: An 8-year observational study. J Allergy Clin Immunol 2004; 114: 1384-8

\section{Antihistaminika im direkten Vergleich}

Es gibt bereits einige Untersuchungen, welche die Wirksamkeit und Sicherheit der $\mathrm{H}_{1}$-Rezeptorenblocker Ebastin und Loratadin bei allergischer Rhinitis verglichen haben. Texanische Forscher wollten jetzt in einer randomisierten Doppelblindstudie an einer großen Fallzahl und mit verschiedenen Dosierungen die Kenntnisse erweitern.

nsges nsgesamt 749 Patienten mit allergischer Rhinitis und durch Pricktest bestätigter Ragweedallergie erhielten während der Pollensaison 4 Wochen lang entweder einmal täglich $10 \mathrm{mg}$ Ebastin, 20 mg Ebastin, 10 mg Loratadin oder Plazebo. Die Wirksamkeit der Medikation erfassten die Patienten dreimal täglich mithilfe eines Symptomscores mit den Parametern laufende Nase, Obstruktion, Juckreiz, Niesen und Augenreizung.

649 Patienten beendeten die Studie. In allen Verumgruppen ergab sich gegenüber der Plazebogruppe eine signifikant reduzierte Rhinitissymptomatik. Während dies im Falle von Ebastin für alle Einzelparameter galt, war der Unterschied zwischen Loratadin und Plazebo nur bezüglich Niesen und Juckreiz signifikant. Beim Vergleich der Wirksamkeit von Ebastin $10 \mathrm{mg} / \mathrm{d}$ mit Loratadin $10 \mathrm{mg} / \mathrm{d}$ zeigte sich außer bei der nasalen Sekretion kein signifikanter Unterschied. Beim Vergleich von Ebastin $20 \mathrm{mg}$ mit Loratadion $10 \mathrm{mg}$ war Ebastin in allen Scores Loratadin signifikant überlegen. Auch die Patienten beurteilten Ebastin $20 \mathrm{mg} / \mathrm{d}$ signifikant besser als Loratadin $10 \mathrm{mg} / \mathrm{d}$. Bezüglich der Sicherheit waren alle vier Gruppen vergleichbar.

Fazit: Diese Studie bestätigt die Wirksamkeit und Sicherheit von Ebastin und Loratadin in der Therapie der allergischen Rhinitis. Im Vergleich zu Plazebo erzielte die Therapie mit 10 oder $20 \mathrm{mg}$ Ebastin pro Tag eine stärkere Sym-

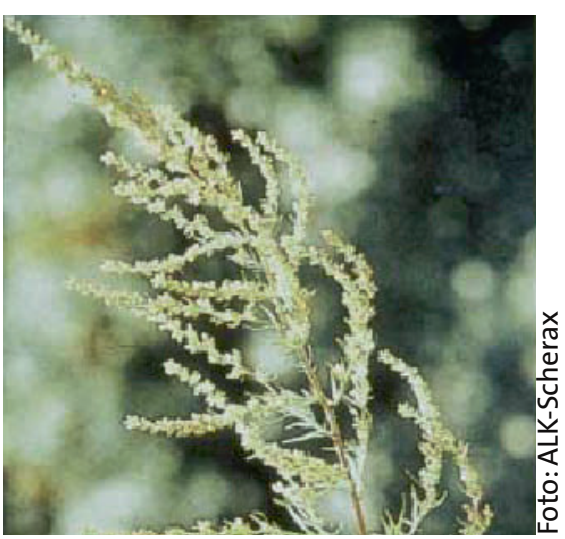

Ein unscheinbarer Allergieauslöser: Ragweed - auch Traubenkraut genannt - breitet sich in Europa zunehmend aus.

ptomreduktion als Loratadin. Ebastin in einer Dosierung von $20 \mathrm{mg}$ vermochte dabei auch die nasale Obstruktion zu verringern.

FK

Hampel $\mathrm{F}$ et al. A randomized, doubleblind, placebo-controlled study comparing the efficacy and safety of ebastine (20 $\mathrm{mg}$ and $10 \mathrm{mg}$ ) to loratadine $10 \mathrm{mg}$ once daily in the treatment of seasonal allergic rhinitis. J Investig Allergol Clin Immunol 2004; 14: 56-63 